\title{
Control design tools for intensified solids handling process concepts*
}

\section{Markku Ohenoja, Marko Paavola and Kauko Leiviskä}

Control Engineering, Faculty of Technology, University of Oulu, Finland

* In book: Advances in Systematic Creativity: Creating and Managing Innovations (2019), eds: L. Chechurin, and M. Collan, Publisher: Palgrave Macmillan, doi: 10.1007/978-3-319-78075-7_11

\section{Introduction}

Theory of inventive problem solving (TRIZ) can be applied to generate new concepts for process intensification. In order to meet the target performance of the intensified process and to avoid design bottlenecks due to process operation, the suggested concept need to comprise a feasible control system. Therefore, a design step, where a systematic procedure for variable selection is performed, available measurement devices are mapped, and the control design is initialized, is needed. This chapter presents a systematic approach to tackle these issues in a structured manner in order to enable a smooth transfer from new innovative ideas into feasible process design from operation point-of-view.

\section{Background}

Process intensification (PI) is process development leading to substantially smaller, cleaner, safer, and more energy efficient technology (Reay et al. 2013). The best known examples come from chemical engineering and miniaturization, such as miniaturized reactors, fuel processing systems, 
power sources and, on the other hand, integrating unit operations such as reactive distillation and dividing-wall columns, see (Baldea 2014; Cremaschi 2015). Indeed, a European research programme has identified that while the highly intensified equipment is largely restricted to gas \liquid and liquid liquid systems also the processes involving solids could benefit especially from continuous mode of operation. However, the intensification of solids handling processes is challenging due to an apparent risk of particulate fouling (scaling, caking, clogging) leading to operational and even safety problems. Taking into account also the complexity of the processes, the intensification project should consider the whole process chain, not only an isolated problem. Intensified by Design ${ }^{\circledR}\left(\mathrm{IbD}^{\circledR}\right)$ platform aims to facilitate a set of design tools aimed for PI and optimization of solids handling processes.

TRIZ can be applied to generate new concepts for PI. As a part of IbD® project, (Livotov et al. 2017) have identified cases where TRIZ and PI methods can be linked efficiently using the platform supported PI database and TRIZ components. According to the Design of Six Sigma (DFSS) approach, the output of the concept development phase needs to lead to a sound, invulnerable and robust system (do the right things, do them all the time) (Yang and El-Haik 2003). As process control is an intrinsic part of process engineering, without a process control assessment, the target of DFSS, and new concepts, is unreachable. The theoretical increment in process efficiency gained through PI might be compromised if the plant is difficult to control and therefore cannot be operated at its nominal operating point (Mauricio-Iglesias et al. 2013). 
The integrated process and control design can be considered as an essential part in transferring a new idea into a product or a process. The integrated process and control design has been reviewed in several studies (Ricardez-Sandoval et al. 2009; Hamid 2011; Yuan et al. 2012; Sharifzadeh 2013; Vega et al. 2014; Huusom 2015). In the intensified processes, the design of process monitoring and control encounters new challenges (Nikačević et al. 2012). Therefore, the integrated design approach is preferable, and it can also lead to novel sensing and actuating solutions in the intensified processes.

In the early stage of control design, it is crucial to identify the aims of process monitoring, which of the monitored variables are to be connected to closed-loop control, should the control be automated, and could the process be optimized during the operation with the proposed control structure. Integrating such an analysis into the process design stage, the intensified process will also have a fit-for-purpose, intensified process monitoring and control solution. Next, a systematic approach is presented to tackle these issues in a structured manner in order to enable a smooth transfer from new innovative ideas into a feasible process design from process operation point-of-view. Finally, the tools are demonstrated with a case study example.

\section{Systematic approach}

Naturally, there exist a number of tools for the integrated design at the early stage. For example, (Hopkins et al. 1998) have presented a design procedure aimed for eliminating all uncontrollable flowsheet designs based on a qualitative structural controllability analysis. The systematic approach 
developed here aims to be an accessible tool for initial process control evaluation starting from information collection. It requires to perform tasks which ensure that all the reasonable interconnecting elements are taken into consideration before proceeding into more detailed design. The approach assists the user to screen and solve the monitoring and control issues of the process design in hand. The systematic approach is divided into three main steps; Initial process analysis, Monitoring advisor, and Control advisor. Here, we focus on Initial process analysis and Control advisor. The flowsheet of the approach is presented in Figure 1. The associated tools are presented in following subsections.

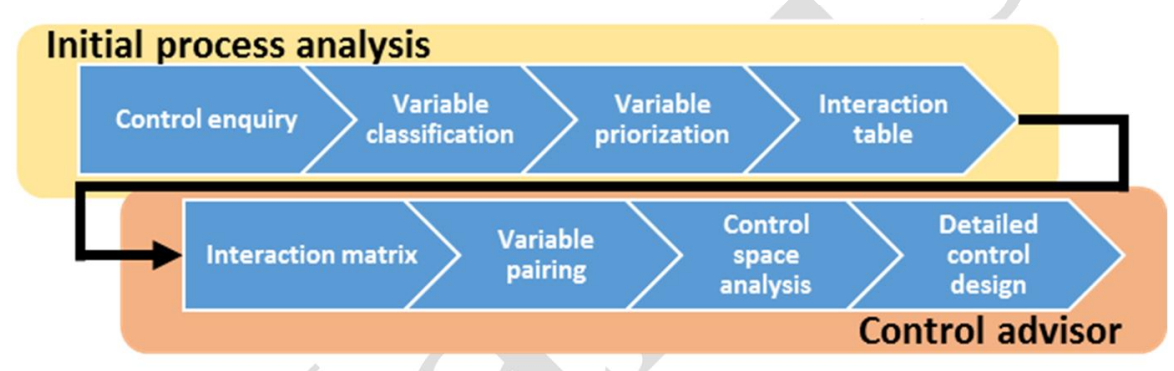

Figure 1. Systematic approach for the initial control design for new process concepts.

\subsection{Initial process analysis}

The initial process analysis step has various objectives. First, the process boundaries and the list of potential monitored variables need to be identified. Then the variables are classified into controlled, manipulated and disturbance variables. Moreover, the critical process parameters and critical 
quality attributes are specified, and the relationship between the selected variables are evaluated. The identification of process boundaries is needed as the control design typically has slightly different boundaries as the process design due to auxiliary equipment, energy and material exchange, and disturbances. This step can be conducted with purely qualitative information and is therefore suitable for being performed for any new process concept. The systematic procedure for the initial process analysis mainly follows the principles of the design steps for the basic control scheme presented in (Roffel and Betlem 2006).

\subsubsection{Control enquiry and variable classification}

The aim of the control enquiry step is to study the operation of the process in a structured manner with the available data such as process flowsheets, PI\&C charts, process operational points, process input (raw materials) and output (product) specifications. The enquiry requires the user to define the goals of operation (target properties of the intermediate products and the final product), and to investigate the process boundaries, as well as the external disturbances. If possible, the process should be divided into independent sub-processes, and the following analysis should be applied separately for every sub-process.

For each sub-process, the controlled (process conditions, material contents, qualities) and manipulated (correcting) variables are defined in variable classification step. Moreover, the process variable(s) that determine the throughput (load) and the recycle flows of the process are specified. 
In addition to the manipulated and controlled variables, variables may also belong to disturbances, observed or not applicablelignored classes. Only one type can be selected for one variable.

\subsubsection{Variable prioritization}

Here, expert knowledge is used for identifying the critical process variables from the comprehensive variable list provided by the previous step. Eventually, a decision is made on which variables are to be considered when the possible monitoring and control solutions are screened. This step can also be executed quantitatively if model-based tools or experimental set-ups are available. In this case, a sensitivity analysis (Saltelli et al. 2006) can be performed and the decision can be based on numerical data. One example of a model-based procedure is presented in (Singh et al. 2009).

\subsubsection{Interaction table}

In the final step of initial process analysis, the identified (most important/sensitive) controlled variables, manipulated variables, and disturbances are arranged into an interaction table for the selected sub-process. Then, a qualitative information of the power and speed of the control between the variables in the interaction table is estimated. The scale is dependent on the fastest/largest response. The interaction table acts as a basis for variable pairing and control design in the Control advisor step.

\subsection{Control advisor}


The control advisor allows to evaluate the desired and available control space with the selected variables, their desired range, and expected disturbances. The control advisor also presents a procedure for process identification, control strategy development, implementation and verification. In order to evaluate the control space, the qualitative information used in the previous step needs to be converted into quantitative information. Naturally, this information is difficult to obtain in the conceptual design phase. The tools can, however, be used also for inaccurate data, allowing the user to inspect the sensitivity of changing process interactions and disturbance space to the design space and control space.

\subsubsection{Interaction matrix}

The interaction table for the selected sub-process generated in the initial process analysis step need to be updated with quantitative information. If quantitative information is not available, or cannot be estimated, the user can use the qualitative interaction table and the guidelines presented in (Roffel and Betlem 2006) for the variable pairing and selection of basic control schemes. If quantitative data are available, the interaction table corresponds an interaction matrix, or a steadystate gain matrix between the selected inputs (manipulated variables, disturbances) and outputs (controlled variables). Also inaccurate estimates can be used. In this case, the matrix represents one possible process expression and the analysis should focus on the sensitivity of different interaction gain estimates to control space and variable pairing. If results are found to be highly sensitive, the 
proposed control strategy does not have generalizable features, and is not feasible for the process concept studied.

\subsubsection{Variable pairing}

The selection of inputs and outputs (IO) in a small system may sometimes be intuitive. The larger the system, the more difficult is the pairing of variables, and performance, complexity and costs of the system may be far from the optimized solution. Systematic methods for variable selection, or input-output (IO) selection, have, for example, reviewed in (van de Wal and de Jager 2001). In the initial phase, a simple criteria based on linear models and IO controllability measures can be recommended. One of the most popular method is the relative gain array (RGA) (Bristol 1966), requiring only a steady-state gain matrix (that is the interaction matrix) as an input, and the RGA matrix as an output. The RGA matrix can then be used with some basic rules to find recommended input-output pairs for univariate control loops. Alternatively, the RGA matrix can indicate the need for multivariate control strategies instead of distributed univariate control strategies.

\subsubsection{Control space analysis and detailed design}

The interactions can be inspected more closely with more sophisticated controllability measures. Due to its visual interface, the output controllability index (OCI) developed in (Vinson and Georgakis 2000) is used here. OCI indicates whether the allowable input space (range of manipulated variables) can satisfy the desired design space (range of controlled variables) with or without the presence of the expected range of disturbances. In this simplest form, the control space 
evaluation is made with steady-state linear models and is therefore well suited for the initial process control design. In the detailed design, the same analysis can be conducted with non-linear and dynamic models (Lima and Georgakis 2010).

The concept phase evaluation is followed by a detailed control design step. It supports, for instance, process identification, control algorithm development, and controller tuning tasks for particular application. In this step, it is necessary to use detailed dynamic simulation models and/or real process environment, and is therefore beyond the concept phase evaluation.

\section{Case example}

The systematic approach described in Section 3 has been applied for a flash flotation cell, a unit process used in an intensified mineral beneficiation process (Newcombe et al. 2013). Table 1 presents a fictitious interaction table with qualitative information for a small scale flash flotation cell. Based on the initial analysis, major disturbances arise from upstream raw material density variations (DV1) and feed flow variations (DV2). One possible control strategy could involve the feed density (CV1) and cell froth depth (CV2) as controlled variables, and water addition rate (MV1) and air addition rate (MV2) as manipulated variables. Ideally, product throughput and quality (mineral concentration and particle size distribution) are controlled, but they cannot be effectively measured online. Table 2 depicts the quantitative strength of interactions, the range of studied variables and their target values in this case example. The analysis is based on uncertain interactions around the nominal and target values of the process. 
Table 1. Qualitative interaction table for the case example.

\begin{tabular}{lll}
\hline & CV1 & CV2 \\
\hline MV1 & Moderate & Small \\
& Fast & Slow \\
MV2 & Nil & Small \\
& & Fast \\
DV1 & Large & Small \\
& Fast & Slow \\
DV2 & Moderate & Small \\
& Fast & Fair \\
\hline
\end{tabular}


Table 2. Quantitative values for the case example. CV1 is the feed density $\left(\mathrm{kg} / \mathrm{m}^{3}\right), \mathrm{CV} 2$ is the froth depth (cm), MV1 is the water addition rate $(\mathrm{L} / \mathrm{h}), \mathrm{MV} 2$ is the air addition rate $(\mathrm{L} / \mathrm{h}), \mathrm{DV} 1$ is the raw material density $\left(\mathrm{kg} / \mathrm{m}^{3}\right)$, and DV2 is the feed flowrate $(\mathrm{L} / \mathrm{h})$.

\begin{tabular}{llllll}
\hline & CV1 & CV2 & Nominal & Minimum & Maximum \\
\hline Target & 1700 & 7 & n.a. & n.a. & n.a. \\
Minimum & 1675 & 6 & n.a. & n.a. & n.a. \\
Maximum & 1725 & 8 & n.a. & n.a. & n.a. \\
MV1 & 0.51 & -0.08 & 90 & 0 & 500 \\
MV2 & 0 & 0.15 & 300 & 260 & 340 \\
DV1 & 0.85 & -0.04 & 1700 & 1600 & 1900 \\
DV2 & 0.71 & -0.10 & 500 & 500 & 700 \\
\hline
\end{tabular}

For the variable pairing, the interaction matrix $\left(\mathrm{G}_{\mathrm{ss}}\right)$ between the manipulated and controlled variables can be written as in Equation 1:

$$
G_{S S}=\left[\begin{array}{cc}
0.51 & 0 \\
-0.08 & 0.15
\end{array}\right]
$$

The corresponding RGA matrix is presented in Equation 2: 
$R G A\left(G_{S S}\right)=\left[\begin{array}{cc}1 & 0 \\ 4 e^{-18} & 1\end{array}\right]$

In the RGA matrix, there is one dominating entry to each column. Hence, the RGA matrix indicates that there are no severe interactions and two univariate control loops could be established; first one between MV1 and CV1, and the second one between MV2 and CV2. From the process engineering point of view, utilizing the water addition rate (MV1) for controlling the feed slurry density (CV1) and adjusting the froth depth (CV2) with the air addition rate (MV2) is reasonable.

For the control space analysis, also the variable ranges and disturbances need to be evaluated. In Figure 2, the control space without accounting for the disturbances is presented. In this case, the control space analysis indicates that the range of MVs is feasible to cover the design space ( $\mathrm{OOCI}=1$ ). It can also be seen that MV1 has unnecessarily large range. In Figure 3, the analysis involves the effect of expected disturbances as well. Clearly, the available control space cannot cover the design space and expected disturbances (oOCI $<1)$. Therefore, the process and control design is not feasible unless the process design is altered. Without the identification of disturbance variables and taking into account their prevailing effect on process behavior, a false justification of the controllability and therefore the feasibility of the proposed process concept could be made. 


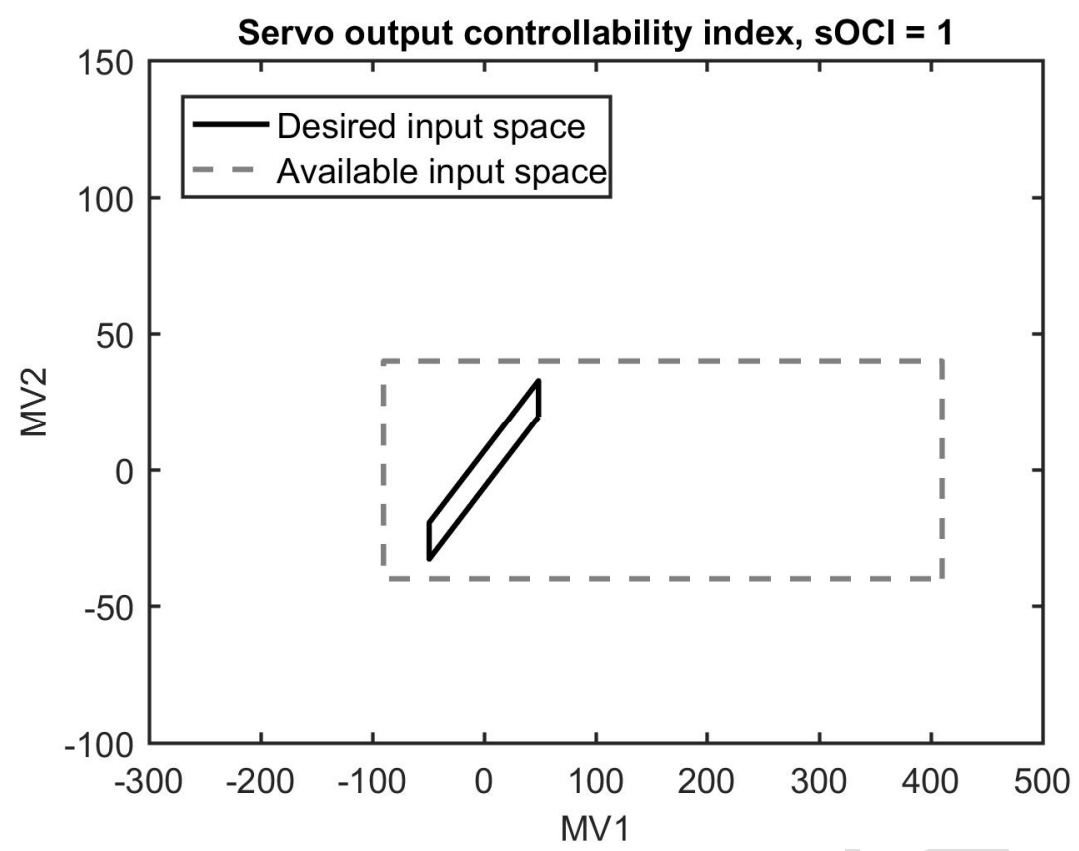

Figure 2. Control space analysis without taking into account the disturbances. MV1 is the water addition rate (around the nominal value of $90 \mathrm{~L} / \mathrm{h}$ ), MV2 is the air addition rate (around the nominal value of $300 \mathrm{~L} / \mathrm{h})$. 


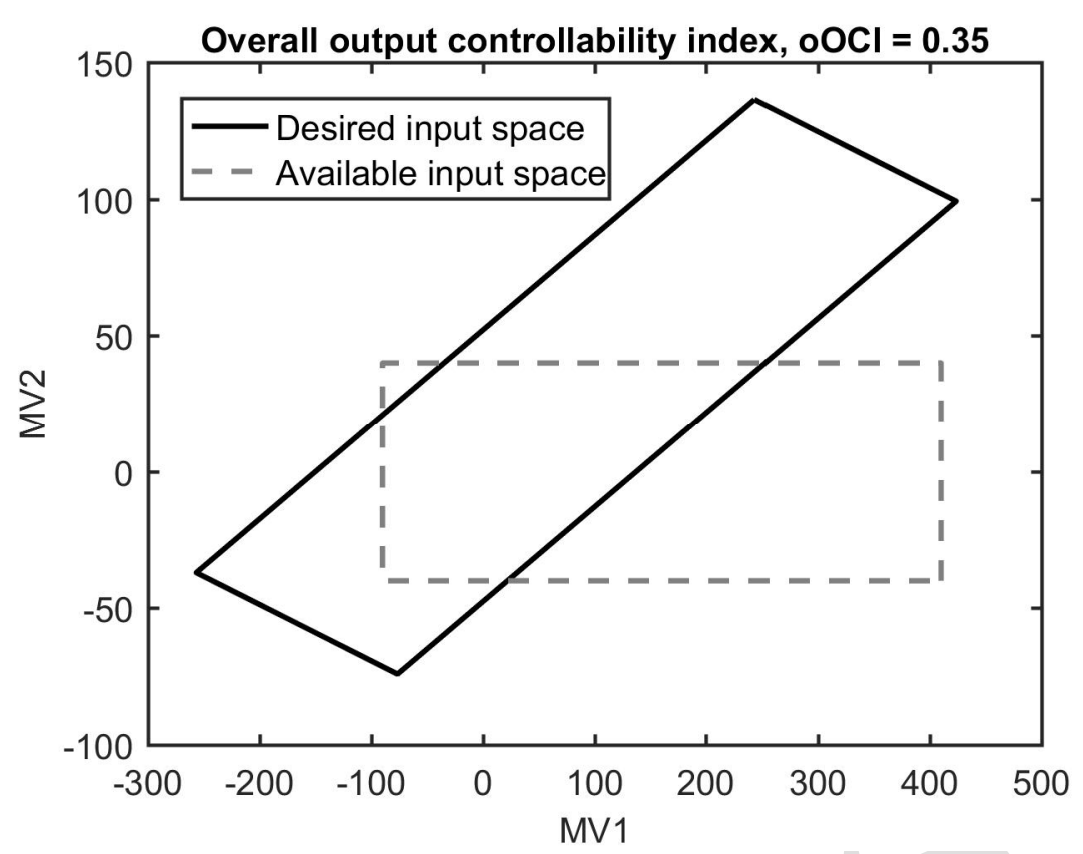

Figure 3. Control space analysis when disturbance variables are taken into account. MV1 is the water addition rate (around the nominal value of $90 \mathrm{~L} / \mathrm{h}$ ), MV2 is the air addition rate (around the nominal value of $300 \mathrm{~L} / \mathrm{h})$.

How can the process design be altered in order to meet the controllability requirements? There are several alternatives to change the process requirements. The first option is to allow more variation in the design space (range of CVs). However, this option is often the last choice or compromise, as the intensification targets cannot be fully achieved. The second option is to expand the ranges of the MVs. It should be noted that there might be physical limitations to the manipulated variables which eventually determine the allowable range. For example, in Figure 3, it can be seen that the range of MV1 should be expanded by setting the lower limit to -280 with respect to the nominal value. 
However, Table 2 depicts that the nominal value is 90 and the current minimum value is zero. Evidently, a negative water addition rate $(\mathrm{L} / \mathrm{h})$ cannot be established and the expansion of MV1 range is not possible in this case. The third option is to control the upstream disturbances by some other means, hence reducing their effect in the inspected sub-process. This approach requires availability and expertise of plant-wide interactions, and may be beyond the process concept evaluation. The fourth option is to discard the proposed set of variables and try a different set of MVs (and possibly CVs). If none of these lead to a controllable process, the process concept studied cannot be seen feasible from the process operation point of view.

\section{Discussion and recommendations}

As presented above, the proposed procedure offers a systematic way for designing control systems for intensified process concepts generated using the TRIZ approach. Together with the tools for choosing the suitable measurements (Monitoring advisor), it will make easier to achieve the feasible monitoring and control solutions in different PI cases. Therefore, for its part, the approach paves way for the industrial adoption of intensified processes. As a limitation, the proposed approach focuses on designing control systems for unit process level. The intensification, in turn, is likely to have an effect on one or more control subsystem(s) consisting of several unit processes, and maybe even plant-wide planning, scheduling and optimization. However, it is an industrial practice to evaluate these aspects during detailed control design and implementation process. In the detailed control design, dynamic behaviour (time-dependency and transitions between different steady- 
states) of the system need to be considered, as well. Dynamic behaviour is an essential feature in control design, but dynamic analyses require detailed models or experimental data, which are likely out of reach during process concept design. Another limitation to the proposed tools is that the control space analysis can efficiently be visualized only to small systems. Larger systems require interpreting several combinations, or evaluating only the numerical controllability measure.

It will be interesting to observe if TRIZ can produce inventive solutions for integrated process and automation solutions as well. The discussion has been initialized in (Chechurin et al. 2016).

Intuitively, the systematic approach presented here could benefit from internal TRIZ exercise targeted to monitoring solutions, for example. With the new monitoring solutions, a different set of variables and therefore new control strategies could be considered. Moreover, the results from this systematic procedure may initialize a need for TRIZ exercise, for instance, to overcome the physical limitations of manipulated variables or to control upstream disturbances.

Acknowledgments The present work has been developed under the financial support received from The EU Framework Programme for Research and Innovation - H2020-SPIRE-2015 (IbD® Intensified by Design. GA - 680565).

\section{References}

Baldea, Michael. 2014. "Multum in Parvo: A Process Intensification Retrospective and Outlook." In Computer Aided Chemical Engineering, edited by John D. Siirola and Gavin P. Towler Mario R. Eden, 34:15-24. Proceedings of the 8th International Conference on Foundations of Computer-Aided Process Design. Elsevier. https://doi.org/10.1016/B978-0-444-634337.50003-1. 
Bristol, E. 1966. "On a New Measure of Interaction for Multivariable Process Control.” IEEE Transactions on Automatic Control 11 (1):133-34. https://doi.org/10.1109/TAC.1966.1098266.

Chechurin, L., V. Berdonosov, and L. Yakovis. 2016. "Heuristic Problems in Automation and Control Design: What Can Be Learnt from TRIZ?" In TRIZ Future Conference 2016. Wroclaw, Poland.

Cremaschi, Selen. 2015. "A Perspective on Process Synthesis: Challenges and Prospects." Computers \& Chemical Engineering, Special Issue: Selected papers from the 8th International Symposium on the Foundations of Computer-Aided Process Design (FOCAPD 2014), July 13-17, 2014, Cle Elum, Washington, USA, 81 (October):130-37. https://doi.org/10.1016/j.compchemeng.2015.05.007.

Hamid, MKA. 2011. "Model-Based Integrated Process Design and Controller Design of Chemical Processes." Ph.D. thesis, Kgs. Lyngby: Department of Chemical and Biochemical Engineering, Technical University of Denmark.

Hopkins, Lisa, Paul Lant, and Bob Newell. 1998. "Output Strutural Controllability: A Tool for Integrated Process Design and Control." Journal of Process Control 8 (1):57-68. https://doi.org/10.1016/S0959-1524(97)00027-9.

Huusom, Jakob Kjøbsted. 2015. "Challenges and Opportunities in Integration of Design and Control." Computers \& Chemical Engineering, Special Issue: Selected papers from the 8th International Symposium on the Foundations of Computer-Aided Process Design (FOCAPD 2014), July 13-17, 2014, Cle Elum, Washington, USA, 81 (October):138-46. https://doi.org/10.1016/j.compchemeng.2015.03.019.

Lima, Fernando V., and Christos Georgakis. 2010. "Input-output Operability of Control Systems: The Steady-State Case.” Journal of Process Control 20 (6):769-76. https://doi.org/10.1016/j.jprocont.2010.04.008.

Livotov, P., A. Chandra Sekeran, Richard Law, Mas'Udah, and David Reay. 2017. "Systematic Innovation in Process Engineering: Linking TRIZ and Process Intensification.” In TRIZ Future Conference 2017. Lappeenranta, Finland.

Mauricio-Iglesias, Miguel, Jakob K. Huusom, and Gürkan Sin. 2013. "Control Assessment for Heat Integrated Systems. An Industrial Case Study for Ethanol Recovery." Chemical Engineering and Processing: Process Intensification, Special issue: Hybrid and Reactive Separations, 67 (May):60-70. https://doi.org/10.1016/j.cep.2012.11.003.

Newcombe, Bianca, D. Bradshaw, and E. Wightman. 2013. "The Hydrodynamics of an Operating Flash Flotation Cell.” Minerals Engineering 41 (February):86-96. https://doi.org/10.1016/j.mineng.2012.11.007.

Nikačević, Nikola M., Adrie E. M. Huesman, Paul M. J. Van den Hof, and Andrzej I. Stankiewicz. 2012. "Opportunities and Challenges for Process Control in Process Intensification." Chemical Engineering and Processing: Process Intensification 52 (February):1-15. https://doi.org/10.1016/j.cep.2011.11.006. 
Reay, D. A., C. Ramshaw, and Adam Harvey. 2013. Process Intensification: Engineering for Efficiency, Sustainability and Flexibility. 2nd edition. Isotopes in Organic Chemistry. Amsterdam ; Boston: Elsevier/BH, Butterworth-Heinemann is an imprint of Elsevier.

Ricardez-Sandoval, L. A., H. M. Budman, and P. L. Douglas. 2009. "Integration of Design and Control for Chemical Processes: A Review of the Literature and Some Recent Results." Annual Reviews in Control 33 (2):158-71. https://doi.org/10.1016/j.arcontrol.2009.06.001.

Roffel, Brian, and B. H. Betlem. 2006. Process Dynamics and Control: Modeling for Control and Prediction. Chichester, England ; Hoboken, NJ: John Wiley \& Sons.

Saltelli, Andrea, Marco Ratto, Stefano Tarantola, and Francesca Campolongo. 2006. "Sensitivity Analysis Practices: Strategies for Model-Based Inference." Reliability Engineering \& System Safety, The Fourth International Conference on Sensitivity Analysis of Model Output (SAMO 2004), 91 (10):1109-25. https://doi.org/10.1016/j.ress.2005.11.014.

Sharifzadeh, M. 2013. "Integration of Process Design and Control: A Review." Chemical Engineering Research and Design 91 (12):2515-49. https://doi.org/10.1016/j.cherd.2013.05.007.

Singh, Ravendra, Krist V. Gernaey, and Rafiqul Gani. 2009. "Model-Based Computer-Aided Framework for Design of Process Monitoring and Analysis Systems." Computers \& Chemical Engineering 33 (1):22-42. https://doi.org/10.1016/j.compchemeng.2008.06.002.

Vega, P., de Rocco Lamanna, S. Revollar, and M. Francisco. 2014. "Integrated Design and Control of Chemical Processes - Part I: Revision and Classification." Computers and Chemical Engineering 71:602-17. https://doi.org/10.1016/j.compchemeng.2014.05.010.

Vinson, David R, and Christos Georgakis. 2000. "A New Measure of Process Output Controllability." Journal of Process Control 10 (2-3):185-94. https://doi.org/10.1016/S0959-1524(99)00045-1.

Wal, Marc van de, and Bram de Jager. 2001. "A Review of Methods for Input/Output Selection." Automatica 37 (4):487-510. https://doi.org/10.1016/S0005-1098(00)00181-3.

Yang, Kai, and Basem El-Haik. 2003. Design for Six Sigma: A Roadmap for Product Development. New York: McGraw-Hill.

Yuan, Z., B. Chen, G. Sin, and R. Gani. 2012. "State-of-the-Art and Progress in the OptimizationBased Simultaneous Design and Control for Chemical Processes." AIChE Journal 58 (6):1640-59. https://doi.org/10.1002/aic.13786. 